Pacific Journal of Mathematics

ARITHMETICAL NOTES. III. CERTAIN EQUALLY 


\section{ARITHMETICAL NOTES, III. CERTAIN EQUALLY DISTRIBUTED SETS OF INTEGERS}

\section{ECKFORD COHEN}

1. Introduction. In this note we shall generalize the following two results in the classical theory of numbers. Let $n$ denote a positive integer with distinct prime divisors $p_{1}, \cdots, p_{m}$,

$$
n=p_{1}^{e_{1}} \cdots p_{m}^{\rho_{m}} \quad(m>0), \quad n=1(m=0),
$$

and place $\Omega(n)=e_{1}+\cdots+e_{m}, \Omega(1)=0$, so that $\Omega(n)$ is the total number of prime divisors of $n$. For real $x \geqq 1$, let $S^{\prime}(x)$ denote the number of square-free numbers $n \leqq x$ such that $\Omega(n)$ is even, and let $S^{\prime \prime}(x)$ denote the number of square-free $n \leqq x$ such that $\Omega(n)$ is odd. It is well-known $[6, \S 161]$ that

$$
S^{\prime}(x) \sim \frac{3 x}{\pi^{2}}, \quad S^{\prime \prime}(x) \sim \frac{3 x}{\pi^{2}} \quad \text { as } x \rightarrow \infty .
$$

Correspondingly, let $T^{\prime}(x)$ denote the total number of integers $n \leqq x$ suct that $\Omega(n)$ is even and $T^{\prime \prime}(x)$ the total number of $n \leqq x$ with $\Omega(n)$ odd. Then $[6, \S 167]$

$$
T^{\prime}(x) \sim \frac{x}{2}, \quad T^{\prime \prime}(x) \sim \frac{x}{2} \quad \text { as } x \rightarrow \infty .
$$

The proof of $(1.2)$ is based upon the deep estimate $[6, \S 155]$ for the Möbius function $\mu(n)$,

$$
M(x) \equiv \sum_{n \leqq x} \mu(n)=o(x),
$$

while the proof of (1.3) is based upon the analogous estimate [6, §167] for Liouville's function $\lambda(n)$,

$$
L(x) \equiv \sum_{n \leqq x} \lambda(n)=o(x) .
$$

In Theorem 3.3 we prove a generalization of (1.2) and in Theorem 3.4 the corresponding generalization of (1.3). The respective proofs are based upon an estimate (Theorem 3.1) corresponding to (1.4) for an appropriate extension of $\mu(n)$ and an estimate (Theorem 3.2) corresponding to (1.5) for the analogous extension of $\lambda(n)$. The proofs of these estimates are in the manner of Delange's proofs $[3, I(b),(c)]$ of (1.4) and (1.5), both being based upon a classical Tauberian theorem (Lemma 3.2) for the Lambert summabillty process. We also require some elementary

Received May 22, 1961. 
estimates contained in $\S 2$, and a lemma on inversion functions (Lemma 2.1).

2. Preliminary results. For an arbitrary set $A$ of positive integers $n$, the characteristic function $a(n)$ and inversion function $b(n)$ of $A$ are defined by

$$
\sum_{d \backslash n} b(d)=a(n) \equiv \begin{cases}1 & (n \in A) \\ 0 & (n \notin A) .\end{cases}
$$

The enumerative function $A(x)$ of $A$ is the number of $n \leqq x$ contained in $A$, and the generating function is the function $f(s)=\sum_{n=1}^{\infty} a(n) / n^{s}$, $s>1$.

We shall be concerned with several special sets of integers. Let $Z$ denote the set of positive integers, $k \in Z$. Then $P_{k}$ will represent the set of $k$ th powers of $Z$, and $Q_{k}$ the set of $k$-free integers of $Z$. The set of $k$-full intergers, that is, the integers (1.1) with each $e_{i} \geqq k$, will be denoted $R_{k}$. We shall use $S_{k}$ to denote the integers (1.1) in which each $e_{i}$ has the value 1 or $k$. Finally, the set of integers (1.1) such that $e_{i} \equiv 0$ or $1(\bmod k), i=1, \cdots, m$, will be denoted $T_{k}$. The characteristic functions $P_{k}, Q_{k}, R_{k}, S_{k}$, and $T_{k}$ will be denoted respectively $p_{k}(n), q_{k}(n), r_{k}(n), s_{k}(n)$, and $t_{k}(n)$; the corresponding enumerative functions will be denoted $P_{k}(x), Q_{k}(x), R_{k}(x), S_{k}(x), T_{k}(x)$. Also let $Q=Q_{2}, Q(x)=$ $Q_{2}(x)$, and $q(n)=q_{2}(n)$. All of the sets defined are understood to include the integer 1 .

REMARK 2.1. It will be observed that $T_{1}=Z, S_{1}=Q_{2}, S_{2}=Q_{3}$.

In addition to the above notation, we shall use $\lambda_{k}(n)$ to denote the inversion function of $P_{k}$ and $\mu_{k}(n)$ the inversion function of $R_{k}$ or $Q_{k}$ according as $k>1$ or $k=1$. By familiar properties of $\mu(n)$ and $\lambda(n)$, [4, Theorem 263 and 300], it follows that

$$
\mu_{1}(n)=\mu(n), \quad \lambda_{2}(n)=\lambda(n) .
$$

LEMMA 2.1. The functions $\mu_{k}(n), \lambda_{k}(n)$ are multiplicative. If $p$ is a prime and $e$ a positive integer, then for $k \geqq 1$,

$$
\mu_{k}\left(p^{e}\right)=\left\{\begin{aligned}
1 & \text { if } e=k \neq 1, \\
-1 & \text { if } e=1 \\
0 & \text { otherwise },
\end{aligned}\right.
$$

while for $k>1$,

$$
\lambda_{k}\left(p^{e}\right)=\left\{\begin{aligned}
1 & \text { if } e \equiv 0(\bmod k), \\
-1 & \text { if } e \equiv 1(\bmod k) \\
0 & \text { otherwise }
\end{aligned}\right.
$$


REMARK 2.2. The multiplicativity property in connection with (2.2) and (2.3) completely determine $\mu_{k}(n), k \geqq 1$, and $\lambda_{k}(n)$ for $k \geqq 2$.

Proof. By definition, if $k>1$,

$$
\sum_{d, n} \mu_{k}(d)=r_{k}(n)=\left\{\begin{array}{ll}
1 & \text { if } n \in R_{k} \\
0 & \text { if } n \notin R_{k}
\end{array} .\right.
$$

Hence, application of the Möbius inversion formula yields

$$
\mu_{k}(n) \sum_{d \mid n} \mu(d) r_{k}\left(\frac{n}{d}\right), \quad k>1 .
$$

Since $\mu(n)$ and $r_{k}(n)$ are multiplicative, it follows by (2.5) that $\mu_{k}(n)$ is also multiplicative (cf. [4, Theorem 265]). Also by $(2.5), \mu_{k}\left(p^{e}\right)=$ $r_{k}\left(p^{e}\right)-r_{k}\left(p^{e-1}\right)$, from which $(2.2)$ results in case $k>1$. The case $k=$ 1 of (2.2) is a consequence of (2.1). The proof of (2.3) is similar and can be omitted.

We recall next some known elementary estimates for $P_{k}(x), Q_{k}(x)$, and $R_{k}(x)$. Let $\zeta(s), s>1$, denote the Riemann $\zeta$-function.

Lemma 2.2. If $k>1$, then

$$
\begin{gathered}
P_{k}(x)=\sqrt[k]{x}+O(1), \\
Q_{k}(x)=\frac{x}{\zeta(k)}+O\left(\frac{k}{\sqrt{x}}\right), \\
R_{k}(x)=c_{k} \sqrt[k]{x}+O\left(\frac{1}{x^{k+1}}\right),
\end{gathered}
$$

where $c_{k}$ is a certain nonzero constant depending upon $k$.

The result (2.6) is trivial, (2.7) is the classical estimate of Gegenbauer (cf. [2, §2]), and (2.8) is a well-known result of Erdös and Szekeres (cf. [1]). In particular, we have

LEMMA 2.3. If $k>1$, then

$$
\begin{array}{ccc}
P_{k}(x) & \sim \sqrt[k]{x}, \quad R_{k}(x) \sim c_{k} \sqrt[k]{\sqrt{x}} \quad \text { as } x \rightarrow \infty, \\
Q_{k}(x) & \sim \frac{x}{\zeta(k)}, \quad\left(Q(x) \sim \frac{6 x}{\pi^{2}}\right) \quad \text { as } x \rightarrow \infty .
\end{array}
$$

We now deduce, for application in $\S 3$, estimates for $S_{k}(x)$ and $T_{k}(x)$ corresponding to those in Lemma 2.3 for $P_{k}(x), Q_{k}(x)$, and $R_{k}(x)$.

LEMmA 2.4. If $k>1$, then 


$$
T_{k}(x) \sim \frac{6 \zeta(k) x}{\pi^{2}} \text { as } x \rightarrow \infty
$$

if $k \geqq 1$, then

$$
S_{k}(x) \sim \frac{6 \alpha_{k} x}{\pi^{2}} \text { as } x \rightarrow \infty
$$

where

$$
\alpha_{k}=\left\{\begin{array}{l}
\zeta(k) \prod_{p}\left(1-\frac{1}{p^{k+1}}+\frac{1}{p^{k+2}}-\cdots-\frac{1}{p^{2 k-1}}\right) \\
\frac{\zeta(2 k)}{\zeta(k)} \prod_{p}\left(1+\frac{2}{p^{k}}-\frac{1}{p^{k+1}}+\frac{1}{p^{k+2}}-\frac{1}{p^{k \neq 3}}+\cdots+\frac{1}{p^{2 k-1}}\right), \\
1,
\end{array}\right.
$$

according as $k$ is even, $k$ is odd and $\neq 1$, or $k=1$, the products ranging over the primes $p$.

REMARK 2.3. It will be noted that $\alpha_{2}=\zeta(2) / \zeta(3)=\pi^{2} / 6 \zeta(3)$.

Proof. The elementary estimate (2.11) was proved in [1, Corollary 2.1]. The result in (2.12), in the cases $k=1$ and $k=2$, is a consequence of (2.10) and Remarks 2.1 and 2.3. To complete the proof of (2.12) one may therefore suppose that $k>2$.

Under this restriction, we consider the generating function $f_{k}(s)$ of $s_{k}(n)$. In particular, if $s>1$, we have (cf. [4, §17.4])

$$
\begin{aligned}
f_{k}(s) & \equiv \sum_{n=1}^{\infty} \frac{s_{k}(n)}{n^{s}}=\prod_{p}\left(1+\frac{1}{p^{s}}+\frac{1}{p^{k s}}\right) \\
& =\prod_{p}\left(1+\frac{1}{p^{s}}\right)\left[1+\frac{1}{p^{k s}}\left(1+\frac{1}{p^{s}}\right)^{-1}\right] .
\end{aligned}
$$

Since

$$
\sum_{p} \frac{1}{p^{k s}}\left(1+\frac{1}{p^{s}}\right)^{-1} \leqq \sum_{p} \frac{1}{p^{k s}} \leqq \sum_{n=1}^{\infty} \frac{1}{n^{k s}}=\zeta(k s), \quad k s>1,
$$

it follows from (2.14) that

$$
f_{k}(s)=\left(\frac{\zeta(s)}{\zeta(2 s)}\right) g_{k}(s), \quad s>1
$$

where

(2.16) $\quad g_{k}(s) \equiv \sum_{n=1}^{\infty} \frac{a_{k}(n)}{n^{s}}=\prod_{p}\left(1+\frac{1}{p^{s k}}-\frac{1}{p^{s(k \neq 1)}}+\cdots\right), \quad s>\frac{1}{k}$,

the product, and hence the series, in (2.16) being absolutely convergent 
for $s>1 / k$. By Dirichlet multiplication [4. \$17.1] one deduces from (2.15) and (2.16) that

$$
s_{k}(n)=\sum_{d \delta=n} q(d) a_{k}(\delta),
$$

because $\zeta(s) / \zeta(2 s)$ is the generating function of $q(n)$, [cf. [4, Theorem 3021). Applying (2.7) in the case $Q(x) \equiv Q_{2}(x)$, it follows that

$$
S_{k}(x) \equiv \sum_{m \leqq x} s_{k}(n)=\sum_{d \lesssim \leqq x} q(d) a_{k}(\delta)=\sum_{n \leqq x} a_{k}(n) Q\left(\frac{x}{n}\right),
$$

and hence that

$$
S_{k}(x)=\frac{6 x}{\pi^{2}} \sum_{n \geqq n} \frac{a_{k}(n)}{n}+O\left(x^{1 / 2} \sum_{n>x} \frac{\left|a_{k}(n)\right|}{n^{1 / 2}}\right) .
$$

Recalling that the series in (2.16) converges absoltutely for $s>1 / k$, one obtains, since $k>2$,

$$
s_{k}(x)=\frac{6 x}{\pi^{2}} \sum_{n=1}^{\infty} \frac{a_{k}(n)}{n}+o\left(x \sum_{n>x} \frac{a_{k}(n)}{n}\right)+o\left(x^{1 / 2}\right),
$$

so that

$$
S_{k}(x)=\frac{6 \beta_{k} x}{\pi^{2}}+o(x), \quad \beta_{k}=g_{k}(1) .
$$

It is readily verified, using (2.16) with $s=1$, that $\beta_{k}=\alpha_{k}$, which completes the proof of (2.12).

3. The principal results. We introduce some further definitions and notation. A divisor $d$ of $n$ will be called unitary if $d \delta=n,(d, \delta)=1$. The function $\Omega^{\prime}(n)$ is defined by $\Omega^{\prime}(n)=\Omega(g)$ where $g$ is the maximal, unitary, square-free divisor of $n$. Let $S_{k}^{\prime}$ and $S_{k}^{\prime \prime}$, denote, respectively, the subsets of $S_{k}$ for which $\Omega^{\prime}(n)$ is even or odd, $n \in S_{k}$. Analogously, let $T_{k}^{\prime}$ and $T_{k}^{\prime \prime}$ denote the respective subsets of $T_{k}$ for which $\Omega(n)$ is even or odd, $n \in T_{k}, k$ even. In addition, we shall use $S_{k}(x), S_{k}^{\prime \prime}(x)$, $T_{k}^{\prime}(x), T_{k}^{\prime \prime}(x)$ to denote the enumerative functions of $S_{k}^{\prime}, S_{k}^{\prime \prime}, T_{k}^{\prime}, T_{k}^{\prime \prime}$, respectively.

REMARK 3.1. It will be observed that $S_{1}^{\prime}(x)=S^{\prime}(x), S_{1}^{\prime \prime}\left(x\left(=S^{\prime \prime}(x)\right.\right.$, $T_{2}^{\prime}(x)=T^{\prime}(x), T_{2}^{\prime \prime}(x)=T^{\prime \prime}(x)$. In addition, we have, by Lemma 2.1, $\mu_{k}(n)=(-1)^{\Omega^{\prime}(n)} s_{k}(n)$, and in case $n$ is even, $\lambda_{k}(n)=(-1)^{\Omega(n)} t_{k}(n)$.

In addition to the lemmas of $\S 2$ we shall need the following three known theorems.

LEMMA 3.1 (cf. $\left[5,259\right.$, p. 449]). For bounded coefficients $a_{n}$, the series, 


$$
\sum_{n=1}^{\infty} a_{n}\left(\frac{x^{n}}{1-x^{n}}\right)
$$

is convergent, provided $|x|<1$.

Lemma $3.2([3, \mathrm{p}, 38])$. If the series

$$
\sum_{n=1}^{\infty} n a_{n}\left(\frac{x^{n}}{1-x^{n}}\right)=S
$$

converges for $0 \leqq x<1$, and

$$
\lim _{x \rightarrow 1^{-}}(1-x) \sum_{n=1}^{\infty} n a_{n}\left(\frac{x^{n}}{1-x^{n}}\right)=S,
$$

then the series $\sum_{n=1}^{\infty} a_{n}$ converges with sum $S$ provided $a_{n}=O(1 / n)$.

LEMMA 3.3 ([7, p. 225]). Suppose that the series $\sum_{n=1}^{\infty} a_{n} x^{n}$ converges for $0 \leqq x<1$ and diverges for $x=1$. If further, $s_{n} \equiv a_{1}+\cdots+a_{n}>0$ for all $n$, and $s_{n} \sim C n(C$ constant $)$ as $n \rightarrow \infty$, then

$$
\lim _{x \rightarrow 1^{-}}(1-x) \sum_{n=1}^{\infty} a_{n} x^{n}=C .
$$

TheoRem 3.1. If $k \geqq 1$, then

$$
M_{k}(x) \equiv \sum_{n \leqq x} \mu_{k}(n)=o(x) .
$$

Proof. By Lemmas 2.1 and 3.1, and the definition of $\mu_{k}(n)$,

$$
\begin{aligned}
\sum_{n=1}^{\infty} \mu_{k}(n)\left(\frac{x^{n}}{1-x^{n}}\right) & =\sum_{n=1}^{\infty} \mu_{k}(n) \sum_{m=1}^{\infty} x^{n m}=\sum_{h=1}^{\infty}\left(\sum_{d, h} \mu_{k}(d)\right) x^{h} \\
& = \begin{cases}\sum_{h=1}^{\infty} r_{k}(h) x^{h}=\sum_{n \in R_{k}} x^{n} & \text { if } k>1, \\
x & \text { if } k=1 .\end{cases}
\end{aligned}
$$

By (2.9), the set $R_{k}$ has density 0; hence Lemma 3.3 with $C=0$ can be applied to the power series so that

$$
\lim _{x \rightarrow 1^{-}}(1-x) \sum_{n=1}^{\infty} \mu_{k}(n)\left(\frac{x^{n}}{1-x^{n}}\right)=0, \quad k \geqq 1 .
$$

Since $\left|\mu_{k}(n)\right| \leqq 1$, Lemma 3.2 is applicable with $a_{n}=\mu_{k}(n) / n$, and one concludes that

$$
\sum_{n=1}^{\infty} \frac{\mu_{k}(n)}{n}=0 .
$$

Put $A_{k}(x) \equiv \sum_{n \leqq x}\left(\mu_{k}(n) / n\right)$; then by partial summation, 


$$
\left.M_{k}(x)=-\sum_{n \leq k} A_{k}(n)+A_{k}(x)([x])+1\right) .
$$

Since $A_{k}(x)=o(1)$ by (3.2), the theorem results from (3.3).

Theorem 3.2. If $k \geqq 2$, then

$$
L_{k}(x) \equiv \sum_{n \leq x} \mu_{l k}(n)=o(x) .
$$

The proof is similar to that of Theorem 3.1 and is therefore omitted. Note that (3.1) reduces to (1.4) in case $k=1$ and that (3.4) to (1.5) in case $k=2$.

Theorem 3.3. If $k \geqq 1$, then

$$
S_{k k}^{\prime}(x) \sim \frac{3 \alpha_{k} x}{\pi^{2}}, \quad S_{k}^{\prime \prime}(x) \sim \frac{3 \alpha_{k} x}{\pi^{2}} \quad \text { as } \quad x \rightarrow \infty,
$$

$\alpha_{l e}$ being defined by (2.13).

Proof. By (2.12), Remark 3.1, and (3.1), one obtains

$$
\begin{aligned}
& S_{h}^{\prime}(x)+S_{k}^{\prime \prime}(x)=S_{k}(x)=\frac{6 \alpha_{k} x}{\pi^{2}}+o(x), \\
& S_{k}^{\prime}(x)-S_{l k}^{\prime \prime}(x)=M_{k}(x)=o(x),
\end{aligned}
$$

and (3.5) results immediately.

Similarly, one may deduce from (2.11), Remark 3.1 and (3.4),

THeOREm 3.4. If $k>1, k$ even, then

$$
T_{k l}^{\prime}(x) \sim \frac{3 \xi(k) x}{\pi^{2}}, \quad T_{k}^{\prime \prime}(x) \sim \frac{3 \xi(k) x}{\pi^{2}} \quad \text { as } \quad x \rightarrow \infty .
$$

Finally, it will be observed that (3.5) becomes (1.2) in case $k=1$; while (3.6) becomes (1.3) when $k=2$.

It is possible to extend (3.6) so as to hold for all $k>1$. Let $g^{*}$ denote the largest unitary divisor of $n \in T_{k}$, such that all prime factors of $g^{*}$ have multiplicity $e \equiv 1(\bmod k)$. Place $\Omega^{*}(n)=\omega\left(g^{*}\right)$, where $\omega(n)$ is the number of distinct prime divisors of $n$, and let $T_{k}^{*}(x)$ and $T_{k}^{* *}(x)$ denote the number of $n \leqq x$ contained in $T_{k}$ according as $\Omega^{*}(n)$ is even or odd, respectively. Then

Theorem $3.4^{\prime}$. If $k>1$,

$$
T_{k}^{*}(x) \sim \frac{3 \xi(k) x}{\pi^{2}}, \quad T_{k}^{* *}(x) \sim \frac{3 \xi(k) x}{\pi^{2}} \quad \text { as } \quad x \rightarrow \infty .
$$




\section{BIBLIOGRAPHY}

1. Eckford Cohen, Arithmetical Notes, II. An estimate of Erdös and Szekeres, to appear in Scripta Mathematica.

2. - Some sets of integers related to the k-free integers, Acta Scientiarum Mathematicarum (Szeged), 22 (1961), 223-233.

3. Hubert Delange, Théorèmes Taubériens et applications arithmétiques, Mémoires de la Société Royale des Sciences de Liège (4), 16, No. 1 (1955).

4. G. H. Hardy and E. M. Wright, Introduction to the Theory of Numbers, 3rd edition, Oxford, 1954.

5. Konrad Knopp, Theory and Application of Infinite Series. 2nd English edition, 1951.

6. Edmund Landau, Handbuch der Lehre von der Vorteilung der Primzahlen, 2 (1909.)

7. E. C. Titchmarsh, The Theory of Functions, 2nd edition, Oxford, 1939.

\section{The UNIVERSity OF TENNESSEE}




\section{PACIFIC JOURNAL OF MATHEMATICS}

\section{EDITORS}

Ralph S. Phillips

Stanford University

Stanford, California

M. G. Arsove

University of Washington

Seattle 5, Washington
A. L. Whiteman

University of Southern Californla

Los Angeles 7, California

Lowell J. Paige

University of California

Los Angeles 24, California

\section{ASSOCIATE EDITORS}

E. F. BECKENBACH

D. DERRY

H. L. ROYDEN

E. G. STRAUS

T. M. CHERRY

M. OHTSUKA

E. SPANIER

F. WOLF

\section{SUPPORTING INSTITUTIONS}

UNIVERSITY OF BRITISH COLUMBIA

STANFORD UNIVERSITY

CALIFORNIA INSTITUTE OF TECHNOLOGY

UNIVERSITY OF CALIFORNIA

MONTANA STATE UNIVERSITY

UNIVERSITY OF TOKYO

UNIVERSITY OF UTAH

UNIVERSITY OF NEVADA

NEW MEXICO STATE UNIVERSITY

OREGON STATE UNIVERSITY

UNIVERSITY OF OREGON

OSAKA UNIVERSITY

WASHINGTON STATE UNIVERSITY

UNIVERSITY OF WASHINGTON

UNIVERSITY OF SOUTHERN CALIFORNIA

AMERICAN MATHEMATICAL SOCIETY CALIFORNIA RESEARCH CORPORATION SPACE TECHNOLOGY LABORATORIES NAVAL ORDNANCE TEST STATION 


\section{Pacific Journal of Mathematics}

\section{Vol. 12, No. $1 \quad$ January, 1962}

Jonathan L. Alperin, Groups with finitely many automorphisms $\ldots \ldots \ldots \ldots \ldots \ldots \ldots \ldots$

Martin Arthur Arkowitz, The generalized Whitehead product ................ 7

John D. Baum, Instability and asymptoticity in toplogical dynamics . . . . . . . . . . 25

William Aaron Beyer, Hausdorff dimension of level sets of some Rademacher series .... $\quad 35$

Frank Herbert Brownell, III, A note on Cook's wave-matrix theorem . . . . . . . . . . . . . 47

Gulbank D. Chakerian, An inequality for closed space curves ................. 53

Inge Futtrup Christensen, Some further extensions of a theorem of Marcinkiewicz ....... 59

Charles Vernon Coffman, Linear differential equations on cones in Banach spaces . . . . . 69

Eckford Cohen, Arithmetical notes. III. Certain equally distributed sets of integers . . . . . 77

John Irving Derr and Angus E. Taylor, Operators of meromorphic type with multiple poles

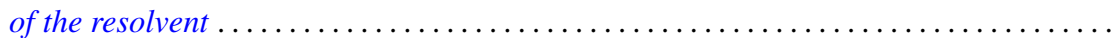

Jacob Feldman, On measurability of stochastic processes in products space .............

Robert S. Freeman, Closed extensions of the Laplace operator determined by a general class of boundary conditions, for unbounded regions ......................

Robert E. Fullerton, Geometric structure of absolute basis systems in a linear topological

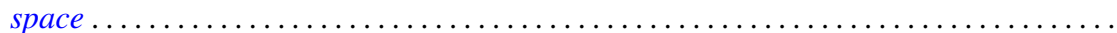

Dieter Gaier, On conformal mapping of nearly circular regions

Andrew Mattei Gleason and Hassler Whitney, The extension of linear functionals defined

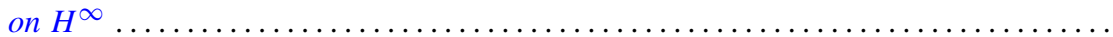

Seymour Goldberg, Closed linear operators and associated continuous linear

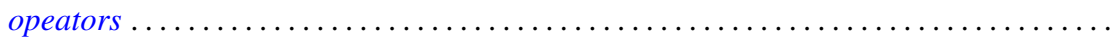

Basil Gordon, Aviezri Siegmund Fraenkel and Ernst Gabor Straus, On the determination of sets by the sets of sums of a certain order

Branko Grünbaum, The dimension of intersections of convex sets. .

Paul Daniel Hill, On the number of pure subgroups

Robert Peter Holten, Generalized Goursat problem . .

Alfred Horn, Eigenvalues of sums of Hermitian matrices ...........

Henry C. Howard, Oscillation and nonoscillation criteria for

$$
y^{\prime \prime}(x)+f(y(x)) p(x)=0
$$

Taqdir Husain, $S$-spaces and the open mapping theorem ...

Richard Eugene Isaac, Markov processes and unique stationary probability measures ...

John Rolfe Isbell, Supercomplete spaces ....................

John Rolfe Isbell, On finite-dimensional uniform spaces. II .........

N. Jacobson, A note on automorphisms of Lie algebras ..............

Antoni A. Kosinski, A theorem on families of acyclic sets and its applications

Marvin David Marcus and H. Minc, The invariance of symmetric functions of singular values...

Ralph David McWilliams, A note on weak sequential convergence.

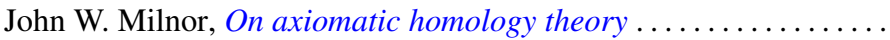

Victor Julius Mizel and Malempati Madhusudana Rao, Nonsymmetric projections in

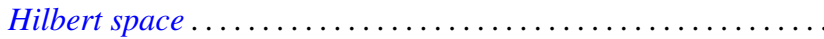

Calvin Cooper Moore, On the Frobenius reciprocity theorem for locally compact

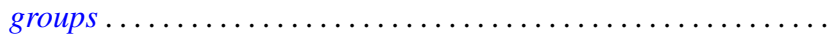

Donald J. Newman, The Gibbs phenomenon for Hausdorff means . 